\title{
LA TEORÍA DEL HABLAR, UNA TEORÍA DEL CONOCIMIENTO
}

\author{
JESÚS G. MARTÍNEZ DEL CASTILLO \\ Universidad de Almería
}

\section{INTRODUCCIÓN}

De las múltiples facetas y consiguientes teorías que Coseriu ve en el fenómeno lenguaje es, sin duda, la teoría del hablar la teoría que estructura y representa a todas ellas. La teoría sobre el cambio lingüístico o de la mutabilidad de las lenguas, la teoría del estudio estructural del significado o lexemática, la lingüística del discurso o lingüística del texto, o las teorías propuestas por Coseriu, como la clasemática o el estudio de los rasgos que estructuran clases léxicas superiores a los campos léxicos, o la esqueuología o el estudio de las relaciones de la lingüística y el conocimiento de las cosas $(1992,148)$, no son más que aspectos parciales de lo que es y significa el hablar en el ser humano, hecho desde el cual el propio Coseriu da nombre a su teoría. La teoría del hablar es y representa la respuesta al problema fundamental y, en términos de Ortega y Gasset, radical del ser humano. Es la teoría del hombre, la teoría del ser que se interroga por sí mismo, que se pregunta qué es ser hombre, qué son las cosas y qué relación mantiene el hombre con las cosas que le rodean. La teoría del hablar es, fundamentalmente, la teoría del ser que se rige por sus propios contenidos de conciencia y que concibe sus elementos como elementos de un acto del conocer.

En este artículo me propongo analizar la teoría de Coseriu como respuesta del hombre a su ser en este mundo, un mundo que él crea dentro de una comunidad de individuos libres, inteligentes e históricos. Desde esta perspectiva la teoría del hablar es una teoría del conocimiento.

Una teoría del conocimiento es aquella que pretende llegar a justificar el conocimiento mismo. Pretende justificar cómo el hombre llega a conocer; pretende determinar qué es y cuál es el sujeto cognoscente, cuál es el objeto conocido y cuál es la realidad tanto del sujeto cognoscente como del objeto conocido. Para llegar a justificar dichos elementos una teoría del conocimiento tiene que explicar la realidad más básica, el propio acto del conocer $\mathrm{y}$, con éste, el propio ser mismo del sujeto que conoce, es decir del hombre; tiene que partir del hombre y, fundándose en el hombre, explicar lo que conoce. Una teoría del conocimiento busca una realidad primera, que ha de ser la más básica, la realidad que dé fundamento a todo lo demás. Por este motivo no puede aceptar nada previamente.

Odisea, $\mathrm{n}^{\circ} 3$, 2003, ISSN 1578-3820, pags. 131-154 
Una teoría del conocimiento tiene su máxima expresión en el significado, aquello que ya de antemano Coseriu define como «contenidos de conciencia» $(1985,27)$. Y ésta es mi tesis en este artículo: la teoría sobre el lenguaje de Coseriu es la respuesta a los interrogantes sobre el hombre y el ser del hombre a partir de la creación constante por éste de contenidos de conciencia o significados.

En el estudio del pensamiento de Coseriu podemos hacer nuestras las palabras de Ortega y Gasset:

El pensamiento de un pensador tiene siempre un subsuelo, un suelo y un adversario. Ninguna de estas tres cosas son lo que [...] está expreso en el pensamiento de un pensador. Queda fuera, y casi nunca el pensador alude a ello. Sin embargo actúa en su pensar y forma parte de él. [...] . Todo texto es fragmento de un contexto inexpreso (1989, 72-73).

Coseriu se propone estudiar el lenguaje, pero su teoría en sí misma es una teoría sobre el hombre y sobre el ser del hombre. Es una teoría del conocimiento implícita, es decir es una filosofía. Coseriu se interesa por todo lo que significa el lenguaje y da respuesta al mismo desde una posición filosófica. Tres son los aspectos de su teoría que me interesa resaltar: el lenguaje como pensamiento o logos, el lenguaje como actividad o enérgeia y el lenguaje como objeto histórico o lengua. Estos tres aspectos son uno mismo en el plano real y la contribución de Coseriu a la lingüística es, precisamente, separarlos, distinguirlos entre sí ${ }^{1}$. Los dos primeros aspectos tienen que ver con el valor universal del lenguaje; el tercero, con la realización histórica del lenguaje, es decir con las lenguas en sus múltiples realizaciones. Junto a estos tres aspectos y para completar su filosofía es interesante también analizar cómo Coseriu desarrolla el estudio de lo que él llama el «conocimiento lingüístico adecuadamente justificado», es decir su estudio científico del lenguaje o lingüística. En la forma de proceder en su propia lingüística se ponen de manifiesto y se completan los elementos de la teoría de conocer implícita.

La teoría de Coseriu, como teoría del conocimiento, se basa en tres pilares fundamentales que el mismo Coseriu plantea en términos de determinación, término que en sí mismo hace alusión a la forma como se concibe algo haciéndolo un ente de razón:

a) determinación del hombre por el hablar;

b) determinación del hablar como actividad, enérgeia; y

c) determinación del hablar históricamente, como lengua.

\footnotetext{
${ }^{1}$ Coseriu concibe el conocer como separar aspectos de un todo indisoluble. En 1985, ante los congresistas de la Modern Humanities Research Association, en Londres, decía: «if I were asked what I consider to be my main contribution to the understanding of language and consequently to the foundation of linguistics, or $[\ldots]$ what constitutes my permanent frame of reference, the very often implicit fundamental principle underlying my treatment of the different, general, or partícular linguistic problems, I would answer that it is a relatively simple distinction, one also made intuitively by the speakers of any language, ....» (Coseriu 1985b, XXV).
}

Odisea, $n^{\circ} 3,2003$ 


\section{LA DETERMINACIÓN DEL HOMBRE POR EL HABLAR}

\subsection{El ser del hombre}

Coseriu se plantea el problema del lenguaje, la teoría del hablar, como el problema del hombre. El hombre en sí mismo es problemático. El lenguaje, igualmente, es problemático porque el lenguaje no es nada sin el hombre. La primera cuestión y el primer fundamento de la teoría del hablar es la solución al problema del hombre. El hombre, para Coseriu, «es el ser que hace de sí mismo un problema y que se pregunta por su propia esencia» $(1985,63)$

Pero el hombre se pregunta por su propia esencia gracias al lenguaje, puesto que lo humano, es decir el ser hombre, comienza en el lenguaje. Coseriu quiere estudiar el lenguaje y tiene que empezar definiendo al hombre, porque el hablar es el compendio del ser humano: «la comprensión del hombre [...] debe comenzar por la comprensión del lenguaje, puesto que lo humano comienza precisamente por el lenguaje» $(1985,63)$.

La esencia del hombre se compendia en su hablar. Hablar para Coseriu es ser hombre, y ser hombre significa preguntarse por sí mismo. Gracias a que el hombre habla se pregunta por sí mismo. La mera pregunta sobre la esencia del hombre implica el lenguaje, porque el lenguaje es hablar y el hablar determina al hombre. Y esto lo afirma Coseriu expresamente: «el lenguaje determina en primer lugar al hombre como tal y lo hace aparecer como hombre» $(1985,63)$.

La primera condición, el primer requisito para responder al problema del lenguaje, pues, es que el hombre sea hombre. Y sólo puede ser hombre si está determinado por el hablar.

Preguntarse el hombre por sí, es decir preguntarse el hombre qué es ser hombre, implica un acto de conocer, implica hablarse a sí mismo sobre lo que es ser hombre, implica concebirse a sí mismo como objeto del conocer y hacerse a sí mismo contenido de su conciencia. Para Coseriu

conocer significa concebir algo como en sí mismo idéntico y como diferente de todo lo demás [...]. Mas aquello que, en el conocimiento lingüístico, se concibe como en sí mismo idéntico y diferente de lo demás no es sino un contenido de conciencia $(1985,27)$.

El hombre llega a saber de sí gracias a su propio acto del conocer, gracias a su propia actividad del pensar. El hombre, que hace de sí mismo un problema, que se pregunta por su propia esencia, llega a saber de sí mismo convirtiéndose en contenido de su propia conciencia. El hombre, así, no es nada que existe de por sí: se hace a sí mismo en su propia conciencia, se hace a sí mismo objeto de su propio conocer. El hombre es desde que se descubre a sí mismo, desde que el hablarse a sí mismo hace que se descubra a sí mismo, desde que el hombre llega a conocerse a sí mismo. Y esto es posible gracias a que el hablar hace posible el conocer. El hombre da respuesta a su propio ser.

Y éste es el fundamento primero de la teoría de Coseriu. El hombre existe porque el hombre llega a conocerse a sí mismo, porque llega a convertirse en objeto de su propio conocimiento. De esta manera para Coseriu el hombre es el único hecho verdadero, el único hecho cierto, el único hecho que puede dar realidad a todo lo demás, tanto a los otros hombres como al mundo y las cosas. El hombre es el punto de partida, la realidad radical que 
diría Ortega y Gasset ${ }^{2}$, la realidad, el dato cierto en el que se ha de apoyar todo el conocimiento posterior. Pero este hecho radical es un hecho complejo. El hombre es hombre por la determinación que ejecuta el lenguaje sobre el mismo. El hombre existe por el lenguaje, y viceversa, el lenguaje no existe si no es en el hombre.

El hombre, hecho hombre preguntándose por sí mismo, haciendo un problema de sí mismo, hecho acto del conocer de sí mismo, se hace hombre, también, preguntándose por el ser, por aquello que le rodea: el hombre es «la capacidad de preguntarse por el ser y de interpretarlo» $(1985,33)$.

Preguntarse por el ser e interpretarlo es concebir el ser como idéntico en sí mismo y como distinto de lo demás, es convertirlo en un contenido de la conciencia del hombre, es convertir el ser en parte del hombre que conoce, es, en definitiva, crear el ser. El ser, las cosas, la realidad, no son de por sí. El ser existe en la conciencia del hombre que se pregunta por el mismo y lo busca en aquello que concibe, haciendo de ello las «cosas». El hombre concibe lo que le rodea interpretándolo de una forma determinada y esa forma determinada de interpretarlo es darle ser, hacerlo existir en su conciencia. El hombre no conoce el ser en sí, puesto que los objetos no tienen ser en sí mismos. El hombre los concibe dándoles y atribuyéndoles el ser. Los objetos sólo son objetos concebidos, contenidos de su conciencia. El hombre en su acto creador crea lo que conoce y conociéndolo, haciéndolo parte de su conciencia, le da el ser y lo ordena. De esta manera las cosas son lo que le atribuimos que sean. Hablamos en esencias, y aplicamos las esencias a lo particular. El mundo que rodea al hombre es un mundo creado, un mundo lingüístico. «El hombre vive en un mundo lingüístico que crea él mismo como ser histórico» $(1985,32)$.

Este dar ser a los objetos es idea fundamental en Coseriu. La teoría del hablar es una teoría del conocer. Es la teoría del crear los elementos que se han de manejar después. Es la teoría que para conocer lo que se aprehende ya convertido en «cosa» ha de crear nuevas cosas. Coseriu concibe al hombre como el ser que aprehende la realidad, el ser que aprehende el ser y lo interpreta, el ser que crea el mundo. El hombre crea el mundo porque conoce y conociéndolo lo hace parte de sí, lo hace parte de su conocer, contenido de su conciencia.

Así, pues, el punto de partida de la teoría de Coseriu es el hombre. El hombre se define por la determinación que el hablar, el lenguaje, ejerce sobre él. Esta determinación es posible gracias a que el hombre conoce. Este es el hecho radical en Coseriu, pero este hecho así formulado es incompleto.

\section{$2.2 \ll$ El ser hablante»}

El hombre y la realidad, vistos por Coseriu, son dos cosas que guardan relación entre sí. El hombre conoce la realidad y la realidad es creada por el hombre. La realidad es así el objeto conocido del hombre. El interés de Coseriu es precisamente explicar esta realidad. Pero entre

\footnotetext{
${ }^{2}$ Para Ortega y Gasset, la realidad última, la realidad en la que se basan todas las demás, el principio estructurador primero, es lo que constituye la realidad radical. Dice Ortega y Gasset: el hombre vive envuelto en «una muchedumbre, no sólo de cosas, sino de modos de ser reales las cosas»; esto «nos obliga a buscar una realidad máxima o realidad radical que nos sirva de unidad de medida para graduar las demás, para ponerla en su sitio y rango de realidad, para jerarquizarlas ontológicamente, para asentarlas y arraigarlas en el ser» (Ortega y Gasset 1996, 40-41).
}

Odisea, $n^{\circ} 3,2003$ 
el hombre y su conocimiento media el lenguaje, por lo que el lenguaje se constituye en el conocer mismo del hombre. El hombre conoce por su lenguaje, el hombre es hombre por su lenguaje. La realidad radical en Coseriu no es meramente el hombre, es el hombre determinado por su hablar: «el hombre es 'un ser hablante', más aún, es 'el ser hablante'» $\rangle^{3}(1985,14)$.

El lenguaje se presenta como lo específico, es decir como lo exclusivo y privativo del hombre. Para un «pensador aristotélico», como a él mismo le gustaría decir, lo específico es aquello que determina al género haciéndolo especie. En el hombre aquello que determina al ser hombre, lo específico, es el hablar.

El hombre se define a sí mismo porque habla y esta nota definitoria es exclusiva del hombre. El hombre agota todo lo que es y constituye el hablar. Coseriu explica esta realidad en términos de determinación, en términos de especificación de una realidad universal a un objeto seleccionado y, por tanto, conocido. El hablar manifiesta al hombre: el lenguaje «pertenece a lo propiamente humano, ya que sólo el hombre es libremente activo» (1985, 23).

Ser hombre, ser humano, no es nada dado por la naturaleza. Es un algo cultural. El hombre es un ser absoluto $(1985,47)$, es decir creador $(1985,97)$, un ser que hace el mundo en el que vive, un ser que va más allá de la experiencia $(1992,226)$. Y esto es posible gracias al lenguaje y gracias a que el lenguaje posibilita el conocimiento del hombre: «La estructuración del mundo mediante el lenguaje no es una limitación, sino la posibilidad misma del conocimiento de este mundo como tal» $(1985,42)$

El hombre, conociendo, crea el mundo en el que vive y conoce porque habla. Su mundo tiene una doble proyección. Es un mundo interior, el mundo de los contenidos de conciencia que él mismo crea, por un lado, y, por otro, es un mundo exterior, el mundo que el hombre ordena y designa gracias a sus propios contenidos de conciencia, gracias a su propio mundo interior. El mundo exterior no es más que lo que el hombre llega a aprehender con el lenguaje: «el hombre [...] aparece como un ser que [...] crea un mundo de significados, que es unidad de conciencia y mundo 'exterior', ‘de razón' y 'cosa'» $(1985,50)$.

El hombre, pues, es un ser creador. El ser mismo del hombre consiste en pensamiento y objeto pensado, contenido de conciencia y objeto conocido. El hombre crea porque conoce y porque se hace a sí mismo con su conocimiento, es decir el hombre se hace as sí mismo culturalmente: «en el hombre lo cultural y la finalidad superan constantemente lo biológico y la necesidad» $(1988,74)$.

El hombre, como «unidad de conciencia y mundo 'exterior', de 'razón y cosa'» es un acto del conocer, con una doble dimensión: es, por un lado, un sujeto cognoscente, un sujeto que aprehende el ser, y es, por otro, el objeto conocido, contenidos de su propia conciencia. Como sujeto cognoscente es objetivación de contenidos de la conciencia, y como objeto conocido es mundo aprehendido. De esta manera el hombre, sus contenidos de conciencia, y el mundo, su objeto aprehendido, son objetos que él mismo crea y, como creados, son objetos que en sí mismos constituyen problema para su propio creador: el hombre es «el ser que es capaz de interpretarse a sí mismo y capaz de interpretar al mundo,

\footnotetext{
${ }^{3}$ Para comprender en toda su extensión esta sencilla expresión hemos de remitirnos a la teoría sobre la determinación de Coseriu de la que hablaremos más adelante. El «humilde» determinante artículo nos dice que el hombre es el único ser que habla. Ser que habla y hombre son la misma cosa.
}

Odisea, $\mathrm{n}^{\circ} 3,2003$ 
que se pregunta por su propio destino y por el sentido del ser de las cosas y del ser en general» $(1985,50)$.

La capacidad de preguntarse por sí mismo y la capacidad de preguntarse por las cosas es la misma. Tanto el hombre en sí como las cosas son hechos inseparables. El hombre, creador de su propio destino, puede interpretarse a sí mismo e interpretar al mundo que le rodea. Y ese problema, ese preguntarse por su destino, no alcanza nunca su interpretación definitiva. Como el lenguaje $\mathrm{e}^{4}$, la interpretación del destino del hombre y la interpretación del ser nunca es una cuestión definitivamente resuelta, sino una pregunta que se hace continuamente: «[s]ólo la reflexión sobre lo dado en el lenguaje y a través del lenguaje [...] puede llevar a la comprensión del ser y de la existencia del hombre» $(1985,56)$

La interpretación de las cosas a través del lenguaje tampoco es definitiva. La interpretación de las cosas depende de la determinación histórica del hablar, que veremos más adelante. Pero por otro lado, la interpretación de las cosas no se agota en la primera aprehensión del ser por el lenguaje. El lenguaje nos permite que el hombre se pregunte, también, por «el ser en general», es decir el lenguaje nos permite que el hombre haga ciencia. El lenguaje es la base de la ciencia por varias razones. En primer lugar, el lenguaje es el punto de unión entre el hombre y el mundo: el lenguaje es la «actividad que se coloca como puente mediador entre la conciencia y el mundo» $(1985,97)$.

En segundo lugar, el lenguaje hace accesibles las cosas a la investigación objetiva: «el mundo de las cosas [...] está dado al hombre, pero sólo a través del mundo de los significados: a través de la configuración lingüística» $(1985,28)$.

La configuración lingüística, o estructuración tradicional de los propios contenidos de conciencia, no es más que el conocimiento de una comunidad de hablantes. La ciencia sólo es posible a través del propio conocimiento humano, es decir a través del lenguaje, a través de lo que el hombre dispone en su conciencia como conocido, que es lo dado en y por el lenguaje (manifiesto éste históricamente en las lenguas). El lenguaje es la actividad primera del conocer, «la primera aprehensión del mundo por parte del hombre» $(1985,64)$.

El lenguaje en sí mismo es ya conocimiento y estructuración de la realidad. Pero es una estructuración interior del hombre, «aprehensión de lo universal en lo individual, objetivación de los contenidos intuitivos de la conciencia» $(1985,206)$. Para Coseriu la relación entre ciencia y lenguaje es la relación que se da entre lo secundario y condicionado con lo primario y condicionante. El lenguaje es lo primario, es decir el lenguaje condiciona a la ciencia. El lenguaje es aquello que hace al hombre, aquello de lo cual el hombre no puede prescindir. La ciencia, por el contrario, no es más que un desarrollo del lenguaje, un desarrollo del conocer humano. De aquí que, en tercer lugar, la ciencia no sea posible sin el lenguaje:

Lo que no es posible sin el lenguaje es la ciencia [...]. El lenguaje es presupuesto de las ciencias porque sólo por su medio puede declararse el qué de un objeto cualquiera y porque sólo con respecto a lo dado lingüísticamente es posible la pregunta acerca del qué de las cosas $(1985,29-30)$.

\footnotetext{
${ }^{4}$ «El lenguaje como algo universal, como categoría humana, es necesariamente hoy el mismo de siempre» (1985, 49); la lengua está «determinada constantemente (y no de una vez por todas) por su función, no está hecha sino que se hace continuamente» $(1988,30)$.
}

Odisea, $\mathrm{n}^{\circ} 3,2003$ 
El lenguaje en sí mismo constituye el punto de partida, el medio y el punto de llegada de la ciencia. El lenguaje es el punto de partida de la ciencia porque ésta parte siempre de lo dado en la configuración lingüística. El lenguaje constituye el medio de la ciencia, porque sólo el lenguaje hace posible declarar el qué de un objeto y preguntar por el qué del mismo. Y el lenguaje es el punto de llegada, porque la ciencia,

emprende una determinación especial del lenguaje como lenguaje técnico, tanto para lo ya dado en el lenguaje, como para lo que ella misma descubre o delimita. Sólo que, para lo uno y para lo otro, la ciencia encuentra en el lenguaje su base y su punto de partida (1985, 30).

Así pues y en resumen, el hombre es hombre por el lenguaje. El hombre se define como una determinación del hablar, que es «el ser hablante». «El ser hablante» es el hecho fundamental, inicial y radical de la teoría de Coseriu. Los demás aspectos son desarrollos del mismo.

Esta era la primera determinación que define la teoría del hablar como una teoría del conocimiento. Pero como dice el propio Coseriu el hablar ha de ser ulteriormente determinado «mediante la determinación de lo que significa propiamente 'hablar'» $(1985,14)$. Dos son las determinaciones del hablar que vamos a ver ahora. En primer lugar el hablar se determina como actividad, y en segundo lugar, el hablar se determina históricamente.

\section{LA DETERMINACIÓN DEL HABLAR COMO ACTIVIDAD, ES DECIR COMO ENÉRGEIA}

Para Coseriu el lenguaje es enérgeia, es decir actividad libre o actividad creadora. Enérgeia es una actividad que por ser libre es una actividad nunca terminada y que por ser creadora es potencia de sí misma. Esta concepción tiene muchas implicaciones de tipo lingüístico, pero nos vamos a fijar en lo que esta concepción implica en sí misma dentro de la teoría del conocimiento.

Para Coseriu el lenguaje como enérgeia es una actividad que deviene en conocimiento. Este tipo de actividad es una actividad libre, expresión que para Coseriu significa actividad creadora (Coseriu 1985,21). Coseriu tiene mucho interés en explicar el sentido de enérgeia y recurre para esto a los creadores de este concepto, a Aristóteles y Humboldt: «Enérgeia es aquella actividad que precede a su potencia, dínamis» $(1992,23)$. Es aquella actividad que causa la imagen de sí misma, que deviene en conocimiento. Es aquello que determina a la actividad en sí haciéndola creativa. En una actividad hemos de distinguir el mero actuar o movimiento, el producto u objeto realizado por la misma y el fin que persigue. Enérgeia es la actividad que supera el mero actuar o movimiento haciéndose potencia de sí misma, es decir conocimiento de sí misma. Es actividad que va más allá de lo actuado, más allá del objeto hecho y del movimiento implícito. Lo actuado no es el fin de la actividad, sino que su fin es infinito:

la actividad anterior a la potencia ( $\delta \dot{v} v \propto \mu \iota c)$, es decir, la actividad creadora o «libre», en el sentido filosófico de la palabra libre. Ahora bien, una actividad «libre» es una actividad cuyo objeto es necesariamente infinito $(1985,21)$.

Odisea, $\mathrm{n}^{\circ} 3,2003$ 
Una actividad, para que sea humana, ha de tener un fin, telos. Para Coseriu, siguiendo con la explicación de Aristóteles, hay dos tipos de actividades: actividades cuyo fin es exterior a las mismas, actividades que se agotan en lo actuado (objeto realizado y movimiento). Éstas, como humanas, implican un conocimiento técnico, y consisten en la aplicación de dicho conocimiento: «actividades productivas que producen algo al aplicar una capacidad de hacer ya adquirida» $(1992,23)$.

Estas actividades se terminan una vez que se han ejecutado, y su dínamis, es decir su conocimiento, es anterior a las mismas. La ejecución de este tipo de actividades no altera en ningún sentido el conocimiento para la ejecución de las mismas. Su finalidad es el ser producido, y por tanto, refieren a un nuevo ser, que en palabras de Ortega y Gasset, es un «ser quieto y estadizo del movimiento que lo ha producido», la entelequia $(1989,193$, nota 35). Estas actividades se dan «cuando producimos ciertos objetos según un modelo formado y con una técnica ya aprendida» (Coseriu 1992, 23).

Pero hay actividades que tienen su fin en sí mismas. Como tales, son actividades nunca llegadas a su perfección, siempre perfectibles, actividades que admiten una ulterior especificación, una ulterior perfección. El conocimiento técnico necesario para la producción de las mismas se convierte en el resultado de su ejecución: «[1] a actividad precede a su dinamis: lo hecho a través de la actividad creadora puede convertirse en un modelo del que se deducen las normas para el hacer» $(1992,23)$.

En contraste con las anteriores, la dínamis, es decir el conocimiento técnico necesario para su ejecución, viene después. Son actividades que parten de sí mismas y llegan a ser conocimiento. Practicadas por segunda vez, parten de un conocimiento anterior y llegan a la perfección del conocimiento inicial; y así en una dialéctica nunca terminada: «[1]o nuevo, lo hecho por primera vez [...] puede ahora, una vez que ha sido hecho, ser tomado [...] como modelo de ulteriores productos» $(1992,23)$.

El conocimiento, de esta manera, es un conocimiento técnico, un conocimiento creativo, un conocimiento que se hace a sí mismo, un conocimiento que sigue a la ejecución de la actividad. Comenta Coseriu que para Aristóteles esta actividad creadora sólo está en Dios en toda su pureza; Coseriu, no obstante, añade: «una actividad humana es creadora en la media en que va más allá de su dínamis y en esa misma medida también el hombre es divino o Dios, como sujeto creador» $(1992,23)$.

Las implicaciones que la aplicación del concepto de enérgeia tiene al hablar o actividad del hablar son muchas. Las que nos interesan resaltar aquí son las siguientes. El lenguaje es actividad creadora: «[e]ntender el lenguaje como enérgeia significa, en consecuencia, considerarlo como actividad creadora en todas sus formas» $(1985,21)$.

El lenguaje o actividad del hablar es actividad creadora tanto en lo que es en sí como en su ejecución. «Enérgeia es tanto el lenguaje en general como el lenguaje en cuanto habla. Todo acto de hablar es [...] un acto creador; de ahí la necesidad de acudir a los contextos y a la situación» $(1985,21)$. También la lengua, el lenguaje como tradición, es actividad creadora, ya que la lengua es un esquema o molde de una actividad: «la lengua tiene existencia concreta como modo formal y semántico de hablar [...]: como forma esquema o molde de una actividad» $(1988,48)$. La lengua es actividad creadora porque se adapta al fin que persigue, es decir la lengua se determina por su fin: «la lengua no 
pertenece al orden causal sino al orden final, a los hechos que se determinan por su función» $(1988,29-30)$.

Por otro lado, la lengua histórica es actividad creadora porque la lengua implica en sí el hablar. La lengua es una parte del hablar y se reconoce en lo constante del hablar: «lo que en el hablar se reconoce como constante y que es objeto de la descripción lingüística» (1992, 91). De esta manera el hablar, la lengua y la descripción de la misma son cosas distintas. El hablar, la actividad del hablar ejecutada en la lengua histórica, es enérgeia, es decir conocimiento lingüístico: «las lenguas [...] existen sólo como modos de hablar que se crean y se continúan como saber lingüístico» $(1988,196)$. La lengua es saber hablar, un conocimiento creativo que es de cada sujeto hablante, que nace y se forma en cada uno de los hablantes, siendo, pues, la lengua nada objetivo en sí misma. «Para cada sujeto hablante la lengua es un saber hablar, el saber cómo se habla en una determinada comunidad y según una determinada tradición» $(1988,57)$.

La creatividad del lenguaje no es nada implícito en el mismo ni en la lengua como objeto histórico, pues el lenguaje está en los hablantes, lo hacen los hablantes, y es nada objetivo. Es algo que pertenece a los hablantes en cuanto que tienen conocimiento lingüístico y lo ejecutan: «sobre la base de este saber, el hablante crea su expresión que, en cuanto coincide con las de otros hablantes o se adopta por ellos, integra (o llega a integrar) la lengua comprobada en el hablar» $(1988,57)$.

El hablante es un sujeto creador y mantiene con el lenguaje una relación dialéctica de creación y objeto creado y con los demás hablantes de creador y destinatario: «todo hablante es creador de lengua 'para otros'» $(1988,57)$.

El lenguaje como enérgeia se extiende a todos los planos del lenguaje: al plano del contenido y al plano de la forma y se manifiesta como acto de significar algo creado por el hablante para el oyente: «la función significativa debe entenderse desde el punto de vista del lenguaje como enérgeia, es decir como actividad creadora» (Coseriu 1985, 26).

Los signos del lenguaje no son nada en sí mismos, son signos porque funcionan y se crean por su función, es decir están determinados por su función:

Los signos lingüísticos organizan formalmente nuestro conocimiento de la realidad, puesto que no son elementos puramente mostrativos sino simbólicos y generalizadores, es decir que no designan individuos, experiencias aisladas, sino que significan géneros, clases, o sea, conceptos generales elaborados por la razón (1986a, 54)

Por otro lado, el lenguaje, que como hemos visto es la aprehensión del ser, se relaciona con la realidad de forma creativa:

Nuestra experiencia de la realidad se elabora mediante las actividades racionales del conocer y del distinguir, y éstas se manifiestan en los signos lingüísticos, por medio de los cuales (a través de sus significados) nos referimos a la actividad extralingüística como algo «sabido» (1986a, 54). 
El lenguaje, la actividad del hablar, la lengua histórica, se resuelven en un acto de conocimiento, en un interpretar la realidad en función del conocimiento (enérgeia), en un crear y distinguir objetos creados (conocimiento, contenido, logos), y en un representar simbólicamente los objetos creados (expresión): «el lenguaje es esencialmente actividad cognoscitiva: una actividad cognoscitiva que se realiza mediante símbolos» $(1985,72)$.

La actividad cognoscitiva que se realiza mediante símbolos es un aprehender la realidad mediante objetos interpuestos, es decir mediante signos lingüísticos que llegan a constituir conocimiento, «forma de conocimiento» $(1985,72)$.

El saber hablar es un conocimiento técnico, es decir un conocimiento seguro, que Coseriu define como cognitio clara confusa, un saber sin justificación, o intuición, cognitio clara distincta inadaequata, saber justificable sólo de forma inmediata (1992, 235). En cualquier caso, una clase de saber seguro, consciente y ejecutable: «el conocimiento lingüístico es un saber seguro y como tal plenamente consciente» (1992, 218). La justificación plena del saber lingüístico es posible en Coseriu, pero ésta se da en el conocimiento reflexivo, en el estudio del lenguaje como saber de los hablantes hecho por los lingüistas.

Esta era la primera determinación del hablar. El hablar se determina como el conocimiento creador, como el «saber» de quien lo crea mediante la ejecución de lo ya sabido y aprendido. El hablar es conocimiento práctico y creador, es decir conocimiento técnico. La determinación del hablar más importante, no obstante, es la determinación histórica.

\section{LA DETERMINACIÓN HISTÓRICA DEL HABLAR.}

El concepto de historicidad es un concepto fundamental en la filosofía desde finales del siglo XIX. Es, si se quiere, el concepto que se va desarrollando, de una manera u otra, en los distintos sistemas de pensamiento sobre el estudio del hombre y de lo humano. En este sentido la contribución de Coseriu hay que ligarla, más o menos directamente, con Dilthey, Heidegger, Ortega y Gasset.

Para Coseriu la historicidad es una determinación del hablar, y es, en este sentido, una característica esencial de la actividad del hablar dada en el «ser hablante». La justificación de la historicidad es dada por Coseriu de forma gráfica: «el lenguaje como hablar es un hablar con otro» $(1985,15)$.

Esta determinación del «ser hablante» define de manera esencial la realidad radical de la que parte Coseriu. El hombre es «el ser hablante» el cual necesariamente habla con otro de tal manera que el hablar no se da más que en el hablar histórico: «el hablar es siempre hablar una lengua» $(1985,16)$.

El ser del hombre es un ser compartido dentro de una comunidad de hablantes, compartir que sólo es posible en la lengua. La condición histórica del hombre dimana del propio ser del hombre: la historicidad es el «modo de ser del hombre, que es un 'ser con otros'» (1988, 43). La justificación de dicho modo de ser está contenida en el hablar mismo:

El hablar, incluso el hablar creador, está dirigido a otros sujetos: la palabra creada está destinada desde el comienzo a valer tanto para el sujeto creador como para los demás sujetos que comparten la misma tradición histórica, pues el sujeto mismo la crea, no como sujeto absoluto [...], sino como miembro de una comunidad idiomática (1985, 48-49). 
«El ser hablante», habla siempre a otro y es siempre un sujeto creador. La creación dentro del hablar es siempre una creación histórica, una creación que está dirigida a otros y que está determinada por su función, el diálogo: «el lenguaje tiene historicidad, y es el fundamento mismo de la historicidad del hombre, porque es diálogo, hablar con otro» y citando a Merleau-Ponty añade: «una conciencia que significa presupone una conciencia que interprete, es decir, que acoja el signo y lo comprenda» $(1988,70)$.

«El ser hablante» es sujeto creador de cosas, significados, e intérprete de lo creado por otros. «El ser hablante» crea el mundo en su conciencia pero lo creado por él no es creación absoluta. La creación humana es siempre intersubjetiva, creación que se da siempre en función de otra dentro de una comunidad de hablantes.

Puesto que el hombre está determinado por el hablar, el hombre está determinado por la propia determinación del hablar. «El ser hablante» es diálogo, ser con otro, ser hablante con otro, ser que se hace a sí mismo creando contenidos de conciencia, haciendo su propia conciencia dentro de una comunidad de hablantes, haciendo su propia conciencia compartida:

La conciencia humana es siempre conciencia histórica, y el modo fundamental de manifestarse en el hombre la conciencia histórica es la «lengua», el hablar como otros, es decir, como ya se ha hablado, de acuerdo con la tradición $(1988,71)$.

Coseriu, a la condición histórica del «ser hablante», llama, también, alteridad, dimensión del hablar que hace al sujeto que habla, al sujeto creador, puesto que éste, en el propio acto de creación, supone a otros:

El lenguaje tiene también otra dimensión, que está dada por la «alteridad» del sujeto, por el hecho de que el sujeto creador del lenguaje presupone otros sujetos, o sea, por ser la conciencia creadora del lenguaje una conciencia abierta hacia otras $(1985,31)$

La historicidad representa el modo de ser del «ser hablante». La historicidad se manifiesta en las lenguas. El hombre como ser histórico tiene un plano determinado de historicidad, es decir pertenece a una comunidad lingüística, habla según una tradición lingüística. El pertenecer a una comunidad significa hablar una misma lengua, tener un mismo y determinado plano de historicidad: encontrarse en un mismo plano de historicidad «no es posible sino por medio de la lengua, que, en el hablante y en el oyente representa su modo de ser histórico» $(1988,70-71)$

Y dado que el hombre es «el ser hablante» la historicidad del hombre es la misma que la historicidad del lenguaje $(1988,69)$. Puesto que «el ser hablante» es histórico, las tradiciones del hablar son históricas, contingentes en sí mismas y distintas unas de otras. La condición histórica es distinta en cada nivel, estructuradora en sí misma y meramente posible: «el lenguaje es la forma expresa e inmediata de la historicidad del hombre; y por la misma razón existen las 'lenguas'» $(1985,49)$.

«El ser hablante» necesita por definición una comunidad, un ámbito en donde se desarrolle como ser histórico. En las lenguas los hombres se reconocen a sí mismos como seres históricos: «[1]a lengua es el terreno común de historicidad lingüística de los hablantes, y 
todo lo que se dice, se dice en una lengua, que, en parte, se manifiesta en forma concreta en lo hablado» $(1982,308)$.

Lo hablado constituye al hombre que habla y el ámbito en donde se desarrolla «el ser hablante» constituye la comunidad lingüística, la cual define al hombre y en virtud de la historicidad del mismo se define a sí misma: «[L]a historicidad peculiar de las lenguas particulares consiste en que constituyen comunidades que se definen a través de ellas» $(1992,98)$.

El ser humano, «ser hablante», ser creativo y libre se realiza en su propia historicidad, es decir en una comunidad lingüística, según una tradición en la técnica del hablar, una tradición en la forma de concebir e interpretar las cosas: «[e]n el hablar histórico, la lengua ya establecida es término necesario de la libertad; pero este término, en cuanto técnica y material para nuevos actos libres, más bien que propiamente 'límite', es condición necesaria de la libertad» $(1988,47)$.

Y en la historicidad se da la intersubjetividad, que tiene un doble sentido: «solidaridad con una tradición histórica y solidaridad 'contemporánea' con una comunidad hablante, que también es histórica» $(1985,32)$. La historicidad es una relación con tres términos: el sujeto creador de la misma, el objeto creado que manifiesta al sujeto creador y el ámbito o grado de la misma o comunidad de hablantes. El sujeto creador de la misma, el sujeto histórico, es «un sujeto que presupone otros sujetos partícipes de la misma historicidad» $(1985,48)$. El sujeto histórico es por definición solidario, es decir partícipe de la historicidad común. La lengua, pues, no le es ajena. Es parte de sí mismo, es el objeto que él moldea.

El objeto creado, el objeto histórico, es el objeto históricamente creado por sujetos históricos que son dentro de una comunidad de hablantes. En tal sentido es algo ya hecho y que se hace al hablar, o mejor: un algo que se hace al hablar basado en modelos anteriores, un algo que lleva en sí lo anterior pero que es nuevo, único y difícilmente repetible. En tal sentido el objeto histórico representa la tradición y la innovación. Es evolución en sí mismo: «[u]n objeto histórico es tal sólo si es, al mismo tiempo, permanencia y sucesión» (1985, 283)

Y el tercer término es un ámbito o grado de la historicidad, que es la comunidad de hablantes, que en sí misma no es más que la lengua que la representa y manifiesta.

\section{EL CONTEXTO HISTÓRICO DE COSERIU}

Coseriu concibe el hablar como una actividad humana universal. Este universal humano es universal del lenguaje, universal empírico y universal lingüístico. Quiere esto decir que para Coseriu el hablar es una cosa que va indefectiblemente con el hombre, en primer lugar; que el hablar se comprueba de hecho en los seres humanos, en segundo lugar; y en último lugar, que el hablar es el principio estructurador de su teoría lingüística. Coseriu parte del hecho, universal en los tres sentidos especificados, único e indivisible, de «el ser hablante». Esta es la originalidad de Coseriu respecto a otros sistemas filosóficos. El lenguaje no es una manifestación de la imaginación o «espíritu del hombre» (Geist) en el intento de dar una respuesta al conocimiento, ni es el lugar en donde el hombre encuentra su esencia, ni es una circunstancia constitutiva del ser del hombre. El lenguaje es la realidad constitutiva de lo que es ser hombre. El hombre no es nada antes del lenguaje. El hombre se hace con el hablar, se determina por el hablar y consiste en el hablar.

Odisea, $n^{\circ} 3,2003$ 
Para ilustrar la contribución de Coseriu en este aspecto podemos compararlo, aunque sea muy someramente, con las posiciones de Humboldt, Heidegger u Ortega y Gasset.

\subsection{Coseriu y Humboldt}

La filosofía anterior a Humboldt estaba dominada por el intento de interpretar el «espíritu» o Geist, objeto por excelencia de la filosofía pura. Humboldt intenta superar la escisión kantiana entre naturaleza y razón. El «espíritu» sólo puede ser aprehendido en la variedad empírica de sus manifestaciones individuales. Para Humboldt lo individual tiene supremacía sobre lo universal. En la investigación filosófica es necesario adoptar una orientación hermenéutica para interpretar lo individual, por un lado, y es necesario, por otro, abrirse al espacio ilimitado de la diversidad, que se despliega entre los dos polos de lo universal y lo individual. Para Humboldt, y es lo nuevo respecto a la filosofía de su tiempo (Di Cesare 1999, 26), lo universal se da únicamente en la variedad de sus manifestaciones individuales. Hace falta, por tanto, una investigación, no tanto trascendental únicamente, sino, al mismo tiempo y a la vez, empírica. La filosofía de Humboldt es la síntesis de lo trascendental y lo empírico, de lo filosófico y lo histórico, de lo posible y lo real (Di Cesare íbidem).

El lenguaje para Humboldt es la manifestación por excelencia del espíritu humano, en la cual se revela la creatividad del género humano en su conjunto. El estudio del hombre remite al estudio del lenguaje, pues este último es la clave para penetrar en la naturaleza humana. La aportación de Humboldt es colocar al lenguaje como centro de la investigación filosófica. Antes de Humboldt, el lenguaje se había considerado como un estorbo dentro de la filosofía. Donatella Di Cesare $(1999,28)$ distingue tres fases en la historia de la filosofía a este respecto. Las edades Antigua y Media se caracterizan por el interés metafísico por el ser y el análisis que se plantean del lenguaje está realizado en función del ser. La Edad Moderna se interesa por la razón que, en la universalidad que se le atribuye, es garantía de una verdad igualmente universal. Considerado en su relación con la razón, en su valor cognoscitivo, el lenguaje se revela el instrumento para expresar esa verdad. Y por último, la fase inaugurada por Humboldt se caracteriza por el interés por el lenguaje sin otra pretensión que el lenguaje en sí. El lenguaje es considerado en sí y por sí, y pensamiento y ser sólo pueden ser considerados en relación al lenguaje, pues son condicionados por el mismo.

Para Humboldt el lenguaje no es instrumento, es órgano ${ }^{5}$, es decir parte misma del hombre. No sirve a nada ajeno a sí mismo, y no es nada en sí fuera del hombre. Nace cada vez como instinto, a partir de una necesidad interior del hombre (Di Cesare 1999, IV).

En tanto que ser natural, el hombre está vinculado originariamente al mundo; en la unidad originaria del hombre y mundo se basa la posibilidad de conocimiento. Esta unidad se rompe con el acto de reflexión en el que el hombre se contrapone a los objetos, determinándose a sí mismo como sujeto. Este acto, necesario para que se construya por una parte el Yo, y por otra el mundo, es un acto lingüístico ${ }^{6}$ : En la reflexión el hombre se contrapone a los objetos y se determina a sí mismo como sujeto.

\footnotetext{
${ }_{5}$ «El lenguaje es el órgano del ser interior, o es este ser mismo tal como poco a poco va abriéndose paso al conocimiento interno y a su manifestación» (Humboldt 1990, 24).

${ }^{6}$ «El lenguaje comienza inmediatamente y al mismo tiempo que el primer acto de la reflexión; [...] se trata [...] del primer estímulo que el hombre se da a sí mismo para detenerse [...] y mirar a su alrededor y orientarse» (Humboldt, apud Di Cesare 1999, 32-33).
}

Odisea, $n^{\circ} 3,2003$ 
Humboldt parte del concepto de conocimiento de Kant. Para éste la sensibilidad y el entendimiento constituyen los dos troncos del conocimiento humano. El conocimiento consiste en la síntesis de ambas facultades, como unificación de la multiplicidad sensible por medio de las formas a priori. En esta síntesis Humboldt introduce la imaginación en tanto que «fuerza creadora», no como una nueva facultad. La imaginación es raíz común a la sensibilidad y al entendimiento, que se escinde de éstas meramente para operar.

El lenguaje no es instrumento para designar objetos ya pensados, sino más bien el órgano formador del pensamiento ${ }^{7}$. La formación de los objetos siempre es subjetiva. La actividad del entendimiento y de los sentidos se combinan sintéticamente para seleccionar, de las impresiones sensibles que acceden al sujeto, los rasgos o los aspectos que caracterizan los objetos para el sujeto y unificarlos mediante una representación subjetiva. La representación subjetiva como tal no es concepto todavía. Para que llegue a ser concepto dicha representación subjetiva tiene que pasar por el tamiz del Tú. Sólo cuando la palabra creada por el Yo es proferida por el Tú, en el diálogo, la representación subjetiva se traduce en objetividad real. El concepto nace tras que la representación subjetiva del Yo sea hecha lenguaje con el Tú, con el otro. Y esta es la intersubjetividad del lenguaje ${ }^{8}$.

De esta manera el Yo no es la subjetividad trascendental, no es el Yo absoluto, ni el individuo abstracto; es el individuo concreto, el individuo inserto en una colectividad, el individuo histórico, el individuo que se define y se hace a sí mismo en diálogo con el Tú, en diálogo con los otros. El individuo en el lenguaje, en el diálogo, se hace a sí mismo, se reconoce a sí mismo como Yo y forma el mundo ${ }^{9}$. Y en la autoformación del Yo y en la formación del mundo a través del diálogo se manifiesta el «trabajo del espíritu», cuya finalidad es la producción del lenguaje. El lenguaje, así, no es tanto una poiesis, relación sujeto-objeto, cuanto una praxis, relación sujeto-sujeto (Di Cesare 1999, cap. IV).

Las diferencias entre la posición de Humboldt y Coseriu se pueden establecer en torno a cuatro puntos principales. En primer lugar el punto de partida, la realidad radical en ambos es distinta. Para Humboldt se trata de justificar el conocimiento, es decir se trata de hacer una filosofía. La imaginación o fuerza del espíritu humano, que dimana tanto de la sensibilidad como del entendimiento y se pone de manifiesto sólo en cuanto que la sensibilidad y el conocimiento se ponen a operar, realiza la síntesis en un acto lingüístico. El lenguaje, pues, está presente desde el principio, pero su presencia es la síntesis de un algo ya dado en el hombre. En Coseriu, por el contrario, la realidad de la que parte, la realidad radical, es «el ser hablante», el ser que es hombre en cuanto que habla, y que no es nada en sí, sino en cuanto que habla. El hablar está determinado, como enérgeia, y como realidad histórica. El hablar así determinado es concreto, tiene existencia concreta. El hombre en sí, sin el hablar, no existe en Coseriu.

En segundo lugar, para Humboldt el lenguaje se realiza en el Tú, en el diálogo, en el hablar con otro. Es el hablar concreto, el hablar empírico, el hablar que se ejecuta en lo individual que, una vez ejecutado, nos revela el lenguaje o lo universal. En lo individual está

\footnotetext{
$\overline{7 \text { «La palabra [...] }}$ se limita a servir de estímulo para producir un concepto, desde la fuerza autónoma de cada uno y de una determinada manera» (Humboldt 1990, 218)

${ }^{8}$ [El lenguaje es] «el gran punto de paso de la subjetividad a la objetividad, de la individualidad siempre limitada a la existencia que incluye en sí todo al mismo tiempo» (Humboldt apud Di Cesare 1999, 39). ${ }^{9}$ «El hombre vive con los objetos de la manera como el lenguaje se los presenta» (Humboldt 1990, 83).
}

Odisea, $n^{\circ} 3,2003$ 
la esencia de lo universal. En Coseriu el hablar es hablar como otro, hablar como ya se ha hablado, hablar como se habla en una comunidad lingüística, hablar según la tradición en la técnica del hablar. En ambos autores el lenguaje es histórico. En Humboldt historicidad es condición concreta, condición empírica del lenguaje (Di Cesare 1999, 34, nota 76) que nos revela lo universal. En Coseriu historicidad es participación, encontrarse ambos partícipes, hablante y oyente, en el mismo nivel de historicidad, hablar y entender lo hablado, hallarse en una comunidad de hablantes. El hablar concreto o individual no nos revela lo universal sino que lo universal, lo histórico y lo individual están presentes en el propio acto del hablar.

De aquí que, en tercer lugar, el lenguaje para Humboldt sea órgano, parte del hombre mismo, la manifestación por excelencia del «espíritu humano», que nace cada vez como instinto, a partir de una necesidad interior. Para Coseriu el hombre es «el ser hablante», el ser que no es si no habla. Lenguaje y humanidad son dos cosas sinónimas. El lenguaje hace al hombre puesto que lo humano se da y empieza en el lenguaje ${ }^{10}$.

Y, por último, en Humboldt lo individual tiene supremacía sobre lo universal, pero individual no es más que una posibilidad de lo universal. Así, la diversidad lingüística, y dado que el lenguaje es órganon, representa una diversidad de visiones del mundo, las cuales nos revelan la verdad. Como unidades individuales respecto a lo universal del lenguaje, las lenguas, cada una en particular, nos revelan el universal, de tal modo que la verdad en su totalidad no está fragmentada en ellas sino que se manifiesta en cada una de ellas. En consecuencia, habría que multiplicar las lenguas para llegar a la verdad (cf. Di Cesare 1999, cap. VII). Para Coseriu el lenguaje es la aprehensión del ser, pero no por parte del individuo absoluto, sino por parte del individuo histórico. El lenguaje no implica verdad alguna de las cosas que designa. El lenguaje coincide con la existencia histórica del hombre, la aprehensión primaria de la realidad, y como tal no es ni verdadero ni falso, pero sí el punto de partida para la verdad contenida en la ciencia.

En definitiva y si valen las reducciones comparativas, Humboldt mira al lenguaje, a lo universal y lo encuentra en las lenguas individuales o históricas que no se realizan en sí mismas sino en el acto del hablar siendo siempre enérgeia. Coseriu mira siempre a la lengua, en cuyos innumerables actos lingüísticos encuentra el conocimiento lingüístico de los hablantes, el cual revela la técnica del hablar, la tradición histórica, el lenguaje y la libertad e inteligencia humanas.

\subsection{Coseriu y Heidegger}

Para Heidegger, el ser es antes que el hombre. El ser es «lo trascendens pura y simplemente. La trascendencia del ser del 'ser ahí' [...] implica la posibilidad y la necesidad de la más radical individuación» (Heidegger 2002, 41).

El ser es aquello que determina a los entes en cuanto entes, aquello en relación con lo cual el ente es comprendido. Pero Heidegger se encuentra con una situación insalvable: el ser no es si no es comprendido, si no es pensado. La solución la encuentra Heidegger en el

\footnotetext{
${ }^{10}$ «La comprensión del hombre [...] debe comenzar por la comprensión del lenguaje, puesto que lo humano comienza precisamente por el lenguaje» (Coseriu 1985, 63). «El lenguaje puede definirse como el primer aparecer - como nacimiento- de lo humano y como apertura de las posibilidades propias del hombre» (Coseriu 1985, 64).
}

Odisea, $n^{\circ} 3,2003$ 
pensar. El pensar produce la relación del ser con la esencia del hombre. El ser, para ser, necesita del pensar, y éste sólo se da en el hombre. El ser solicita ser del hombre, de tal manera que el hombre sólo se deja ser en su esencia en la que es solicitado por el ser. Sólo desde esta solicitud «ha» encontrado el hombre aquello en donde mora su esencia. La esencia del hombre mora en el lenguaje: en el pensar el ser viene al lenguaje. El lenguaje es la casa del ser. En su vivienda mora el hombre. El lenguaje es la vivienda que conserva lo «ecstático» para la esencia del hombre. Desde esta perspectiva y de esta manera, la definición del hombre se ha de hacer en términos de su esencia. Y esta esencia del hombre es la esencia en la que es solicitado por el ser. A esto Heidegger llama la ec-sistencia del hombre, que consiste en «estar en la iluminación del ser». La ec-sistencia es el fundamento de la posibilidad de la razón, ratio, y es aquello donde la esencia del hombre conserva la proveniencia de su determinación (Heidegger 1970, 19)

La ec-sistencia del hombre es histórica, pero no primariamente y sólo porque con el hombre y con las cosas humanas suceden muchas cosas en el transcurso del tiempo (Heidegger 1970, 33). En Heidegger el hombre es llamado a ser por el ser, y es en la medida en que es solicitado por el ser. En este sentido el hombre es determinado por el ser. El ser es dado en el lenguaje, y puesto que el hombre se desarrolla en el tiempo, su esencia o ecsistencia es histórica. En Coseriu, como hemos visto, el hombre no es sin el hablar o lenguaje. El ser es creado en un acto de conocer y es dado en el lenguaje históricamente. Y en esto, en la historicidad del hombre y, por ende, del lenguaje tenemos un punto de coincidencia en el pensamiento de ambos pensadores.

\subsection{Coseriu y Ortega y Gasset.}

Ortega y Gasset estudia al hombre en sí y sin conexión primera con el lenguaje. Para Ortega el hombre es «el ente que se hace a sí mismo» $(1971,42)$, el ser que consiste en ser «lo que aún no es» $(2002,49)$. El hombre se define en función de su propio coexistencia con el mundo, lo que constituye su vivir: «el hombre no es cosa ninguna, sino un drama - su vida un puro y universal acontecimiento que acontece a cada cual y en que cada cual no es, a su vez, sino acontecimiento-》 $(1971,41)$.

Como para Coseriu, el hombre crea la realidad en la que se mueve: «el hombre no encuentra cosas, sino que las pone o supone» $(1971,41)$. El hombre es el ser que se hace a sí mismo en virtud de las decisiones que tiene que tomar en todo momento. En este sentido el hombre es inteligente y libre $(2002,106)$. El hombre es ser histórico, y se hace en la historicidad. El lenguaje es un desarrollo del ser histórico, que lo hace ser social. «La lengua [...] es lo que la gente dice, es el ingente sistema de usos verbales establecidos en una colectividad» $(2001,252)$. Con la lengua penetra la gente en el individuo haciendo de cada cual un caso de «gente». La lengua materna socializa lo más mínimo de nuestro ser, y merced a ello todo pertenece, en el sentido más fuerte del término, a una sociedad.

Para Coseriu el lenguaje es la realidad consustancial del hombre. El hombre no es hombre si no se considera el lenguaje. Más aun: no tiene ningún sentido hablar del hombre si no se concibe éste con el lenguaje. El lenguaje define al hombre y es parte de la definición del hombre. Tampoco existe el lenguaje en sí. La realidad última y radical es «el ser hablante». Más allá de esa realidad no hay nada.

Odisea, $n^{\circ} 3,2003$ 
La coincidencia, de nuevo, entre ambos pensadores está en la historicidad, pero el desarrollo de este concepto en cada uno de ellos es distinto. En Ortega y Gasset ser histórico significa ser heredero de una colectividad: «ser hombre es irremediablemente serlo según el modo o módulo creado milenariamente por una colectividad» (1996, 160). El hombre se encuentra con una realidad dada, la realidad histórica manifiesta en la sociedad. Como ser que tiene que hacerse en lucha contra la circunstancia, la realidad histórica es el medio que le ayuda en dicha lucha, constituyéndose así en heredero de la misma (Ortega y Gasset 1997, 45). La historicidad es la existente en la colectividad, gracias a la cual se ha formado la sociedad y con ella los individuos que se han encontrado con ella. Al individuo, es decir al «hombre», le es dada la vida y éste tiene que elegir y hacerse su propia vida. Para Coseriu ser histórico significa ser que participa con otro en un mismo grado de historicidad (1985, 48). El «ser hablante» es el agente que crea sus propias formas comunes, su propia historicidad con otros. Historicidad es participar, es hacer común lo que en principio como creación es individual. Mediante la participación la creación individual llega a hacerse común, es decir tradicional, es decir histórica. Si bien en Ortega y Gasset «el hombre» tiene que hacerse a sí mismo, se hace a sí mismo en lo histórico. El hombre se hace a sí mismo eligiendo formas ya dadas, formas ya inventadas, formas ya hechas en la colectividad, formas que funcionan y que posibilitan la lucha del hombre con la circunstancia.

La forma de proceder en ambos pensadores es distinta. Coseriu parte del hablante como realidad radical que se manifiesta de forma individual. El individuo, es decir «el hablante», es el único ser existente. A partir de él Coseriu concibe el lenguaje y la lengua. El individuo es el agente que crea el lenguaje, que crea la forma implícita de pensar que lleva la lengua, que crea formas, contenidos y procedimientos. El individuo crea y lo ofrece a los demás en una relación recíproca entre el hablante y «el otro». El hombre es ser con otro, crea y acepta formas para y de los demás, haciéndolas de esta manera comunes, tradicionales, históricas. $\mathrm{Y}$ en este crear, ofrecer y hacer comunes Coseriu ve el fundamento de toda su teoría. La descripción del lenguaje y su manifestación histórica las lenguas se ha de hacer a partir de esta realidad. El lingüista para comprender el lenguaje tiene que abstraer, tiene que partir del individuo y de sus manifestaciones concretas, los actos lingüísticos; tiene que despreciar lo individual y lo particular seleccionando lo que pertenece a la esencia de lo que es y constituye «el ser hablante», por un lado, y seleccionando lo que es común dentro de una tradición del hablar, por otro. Para Coseriu el individuo, que es creador para los demás, con su actividad del hablar se convierte en el creador del lenguaje y de la técnica que lleva implícita el lenguaje. Coseriu, pues, procede inductivamente a partir de la realidad radical de la que parte.

Ortega y Gasset, por el contrario, permanece siempre en el plano deductivo desde la realidad radical de la que parte. La lengua no es más que un conjunto de usos sociales, una cosa derivada de la situación radical y de la condición social e histórica del ser humano. Para Ortega y Gasset la realidad histórica, la sociedad y la lengua, constituyen la circunstancia con la que se encuentra el «hombre». Éste es lanzado a la vida y tiene que hacer algo. Él, por un lado, y esa realidad descrita o circunstancia, por otro, constituyen su «vida», en la que ha de hacerse como ser humano. Ortega y Gasset ve el lenguaje sólo como realidad histórica, como conjunto de usos sociales con los que se encuentra el individuo, «el hombre». 
Basados en la distinta concepción sobre la realidad radical, sobre «el hombre» en Ortega y Gasset o sobre «el ser hablante» en Coseriu, el concepto de logos en uno u otro es distinto. Para Coseriu el logos es el hó formado con el tú en una comunidad lingüística, que representa lo común entre los hablantes y que, siendo común, constituye un acervo lingüístico de formas de concebir y ver la realidad existente en la tradición. Para Ortega y Gasset el lógos es lo que se dice, el «decir», que «es poner de manifiesto lo que antes existía en forma latente y larvada» (Ortega y Gasset 1999, 100). Para Coseriu el logos se crea en la historicidad por los hablantes que son siempre individuos; para Ortega y Gasset el logos existe en la sociedad histórica y el individuo, «el hombre», porta en sí mismo como heredero las formas y pensamientos que lo constituyen. Coseriu parte siempre del individuo que crea; Ortega y Gasset del hombre que porta en sí una «consistencia» histórica, una esencia histórica que metafísicamente es igual a la de todos los hombres.

El tratamiento que cada uno de estos dos pensadores da al lenguaje es, por consiguiente, distinto. Ortega y Gasset concibe el lenguaje en sí, como realidad que es tanto humana como histórica, incluso como una realidad única, una realidad que porta en sí el lógos, el decir y el pensar acumulado durante milenios. Para Coseriu el lenguaje es una realidad universal, el hablar, que se manifiesta individualmente como hablar individualmente, e históricamente como lengua.

De esta manera, el interés de ambos pensadores es opuesto. Ortega y Gasset, como filósofo, procede siempre desde el estado de cosas en cuestión «hacia abajo» o «hacia atrás». Quiere llegar siempre a lo más básico, a lo más profundo, al fundamento último, ya se trate de las verdades en sí, o del pensar de «un pensador» en particular. De aquí que la etimología de las palabras sea la clave del estudio del pensamiento o lógos. Ortega, como Heidegger, utiliza «el lenguaje» (lenguaje y lengua histórica) como el gran medio en el que se manifiesta el pensamiento histórico. Ortega y Gasset procede hacia atrás, hacia la etimología última. Las palabras, dice, «tienen un sentido privilegiado, máximo o auténtico; a saber: el que significaron cuando fueron creadas» (Ortega y Gasset 2002, 124). La razón de esto es que las palabras son la respuesta a una situación vital.

Para Coseriu este planteamiento no es posible, precisamente, por exigencias del hablar y de la historicidad del mismo. La historicidad se estructura sincrónicamente. Cada estado sincrónico es una unidad independiente del siguiente y del anterior. Un estado de la lengua es reconstitución del estado anterior, en parte, igual y, en parte, nuevo. Entre los estados de la lengua se da la solidaridad histórica, es decir la evolución diacrónica, nunca la homogeneidad. Cada estado de la lengua es objeto de estudio en sí mismo. El estudio de un nivel de lengua se detiene en cada estado de la lengua. Por otro lado, las palabras, son objeto de interés para Coseriu en cuanto que son «palabras lexemáticas», es decir significados, es decir contenidos de conciencia. Como tales contenidos de conciencia son individuales e históricas, y constituyen el pensamiento o hó yo Dentro de cada estado histórico, dentro de cada estado de la lengua, los significados,

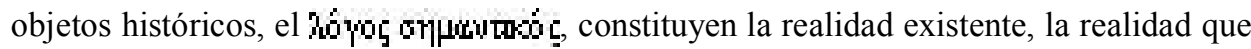
hay que describir, la realidad que ya no es individual ni universal, la realidad que pertenece a la «comunidad». Los significados son elementos comunes, extrasubjetivos, cuya descrip-

Odisea, $\mathrm{n}^{\circ} 3,2003$ 
ción se consigue por abstracción de lo individual y particular y pertenecen al sistema de la lengua.

Los significados son en sí mismos un algo, una cosa objetiva dada históricamente. Para Coseriu, «hay que explicar las cosas como son» (1987). Las cosas son porque son objetos históricos, entes de razón creados por los hablantes como sujetos cognoscentes como los contenidos de conciencia de ellos mismos dentro de una comunidad histórica y en un nivel histórico de la misma. La justificación de la existencia de las «cosas» para Coseriu está dada históricamente. Las cosas existen porque así lo consideran los miembros de una misma historicidad; existen de la forma y en el sentido en que ellos las conciben manifiestas en los significados de la lengua histórica. Dada la variedad lingüística las cosas no son de la misma manera en cada comunidad histórica ni en cada estado de una misma lengua histórica. Todas ellas, sin embargo, se fundamentan en la convención y justificación históricas, אứ ovv8ijor (Coseriu 1977, 13-59). Para Ortega y Gasset las cosas no son nada, son sólo «prágmata».

Por último, y dado que las cosas existen históricamente, el conocer es separar aspectos de las mismas, es decir crear nuevas cosas. Conocer una cosa, formar un concepto basado en la realidad, no es abstraer los puntos comunes de una clase, sino una aplicación a un algo real de una clase previamente concebida $(1992,226)$, aplicación que se realiza sobre la base de una «intuición inmediata de [un] modo de ser» (1986b, 54). Para Ortega y Gasset conocer es vivir en el exterior, salir fuera de sí para que se hagan presentes las cosas buscando en ellas el ser de las mismas $(1992,144)$. Las «cosas» no son nada, son «prágmata», lo que «constituyen» a cada cual. Por esto las cosas no tienen esencia, sino «consistencia».

\section{ELEMENTOS DE LA TEORÍA DEL CONOCIMIENTO}

Para Coseriu hablar es un acto cognoscitivo. Hablar es aprehender el ser e interpretarlo, y consiste en designar un trozo de la realidad (seleccionar un conjunto de las sensaciones que nos llegan a través de los sentidos), atribuirle una esencia (sistematizarlo según patrones individuales o comunes), representarlo simbólicamente (interponer un contenido de conciencia entre hablante y oyente), darle un nombre (atribuirle un signo histórico), orientarlo hacia las cosas (determinarlo), y sobre todo, ofrecerlo a los demás (hacerlo tradicional o histórico en el diálogo con el otro).

\subsection{EI acto lingüístico}

El acto del hablar y del conocer se ejecuta en el acto lingüístico. Para Coseriu un acto lingüístico,

es al mismo tiempo, un hecho individual y un hecho social: hecho individual, en cuanto el individuo hablante expresa de una manera inédita una intuición inédita que le pertenece exclusivamente; $y$ un hecho social en cuanto el individuo no crea íntegramente su expresión sino que más bien la re-crea de acuerdo con modelos anteriores (1986a, 31). 
El acto lingüístico es una intuición inédita que pertenece exclusivamente al sujeto hablante, al sujeto que para hablar conoce, al sujeto que libremente y sin trabas a su aprehensión del ser lo estructura y sistematiza como mejor le conviene, al sujeto que ejercita una actividad libre y actividad creadora cuyo fin es infinito. El acto lingüístico es un acto de creación cognoscitiva: «[s]iendo siempre expresión de una intuición inédita y única, el acto lingüístico es acto de creación (1986a, 27-28).

Es un acto elaborado cognoscitivamente, intelectivamente por el individuo hablante:

acto singular que no reproduce exactamente ningún acto lingüístico anterior y que sólo por los límites que le impone la necesidad de la intercomunicación social se «parece» a actos lingüísticos anteriores, pertenecientes a la experiencia de una comunidad (1986a, 27).

El acto lingüístico es un acto realizado por el individuo en el interior del mismo, en su conciencia. Es un acto que mira a los demás y que implica un acto semejante en el oyente. Es un acto que es realizado por un sujeto que es capaz de realizarlo, de crear en su propia conciencia, un sujeto que es capaz de conocer. El acto lingüístico es tan autónomo en sí como acto cognoscitivo que todo acto lingüístico supera a la lengua:

La «lengua» no nos es nunca enteramente suficiente para expresarnos en cada caso particular, dado que nuestras intuiciones (el contenido cognoscitivo al que tenemos que dar forma de lenguaje) no son nunca idénticas a otras anteriores $(1985,75)$

Para Coseriu el acto lingüístico es más que la lengua y más que el lenguaje; es el conocimiento mismo, el compendio de todo lo que es el hombre. Hasta tal punto el acto lingüístico es el puro acto del conocer que su creación trasciende no sólo a la lengua materna, sino, incluso, a la lengua aprendida: «[h] asta quien habla un idioma distinto del propio es creador, es decir que lo es también en la lengua 'aprendida'» (1985, 76-77).

El conocer es individual y se ejercita en el lenguaje, en el acto lingüístico. El acto lingüístico es el acto del conocimiento y constituye el soporte de toda actividad humana, que se manifiesta en el lenguaje. Éste es el primer elemento que me interesa resaltar como elemento del conocer en la teoría de Coseriu. El acto lingüístico implica una serie de operaciones cognoscitivas, que son lingüísticas y que Coseriu explica como conocimiento de los hablantes.

\subsection{La estructuración de los elementos del hablar}

Entre éstas voy a señalar dos: la propia estructuración que hace Coseriu de los elementos del hablar y el conjunto de operaciones que Coseriu enclava en la determinación.

La configuración del hablar no es más que un ver la misma realidad desde puntos de vista distintos. Para Coseriu conocer es separar aspectos de aquello que en el plano real aparece como indisoluble e informe. No me voy a detener en esto, como ilustración voy sólo a comentar la estructuración del contenido lingüístico.

El contenido lingüístico tal y como lo formula Coseriu es la aplicación de una teoría del conocimiento. Coseriu concibe el contenido lingüístico como estructurado en un triple 
plano. El contenido lingüístico es, en primer lugar, designación, es decir selección de un aspecto del continuum de lo percibido. En segundo lugar es significado, sistematización de los hechos seleccionados, sistematización que se realiza en el plano de lo conocido o de la conciencia. De aquí que el significado no sea más que contenidos de conciencia, como hemos visto. Y en tercer lugar es sentido, la utilización individual por parte del hablante de lo seleccionado y sistematizado, de lo real convertido en contenido de conciencia, es decir es la formulación de la intención significativa de quien habla.

La designación, que se da en el discurso y aparece por tanto en el acto lingüístico, es una operación totalmente individual y es un acto de conocer, de interpretar libremente: «La designación es siempre (aun cuando parece ser automática) interpretación de una 'cosa' con respecto a un significado; y cada cosa se introduce en el molde que mejor se adapta» $(1990,259)$.

El significado es también una operación individual, creativa y cognoscitiva. El lenguaje mismo se define por su función interior, que no es otra que la creación de significados $(1985,26)$.

Y el sentido, que nunca se ha de confundir con lo histórico, es pura creación individual, puro acto del conocer y del hacer el mundo para los demás, incluso, puro acto del distorsionar libremente la realidad a conveniencia de quien habla, de quien conoce. La explicación que da Coseriu de la metáfora es una explicación del sentido en sus últimas consecuencias:

expresión unitaria, espontánea e inmediata [...] de una visión, de una intuición poética, que puede implicar una identificación momentánea de objetos distintos [...] o una hiperbolización de un aspecto particular del objeto [...] y hasta una identificación entre contrarios, lógicamente «absurda», pero de significado y efecto irónicos evidentes, en situaciones determinadas $(1985,81)^{11}$.

\subsection{Proceso de abstracción ascendente y descendente}

El acto del conocer en Coseriu es un proceso ascendente de abstracción de lo real o de elaboración de contenidos necesariamente abstractos. Se parte de la aprehensión, de la sensación, de lo concreto, y se llega a la elaboración de un algo que ya, una vez elaborado, no responde a lo que percibimos, a lo que aprehendimos inicialmente. De esta manera no hablamos de lo real, sino de esencias. Aplicamos esencias a aquello que seleccionamos, a aquello que designamos y de lo que queremos hablar. Es un proceso de abstracción ascendente. Pero para referirnos a la cosa concreta que intentamos tenemos que hacer un proceso cognoscitivo inverso. Necesitamos orientar lo que es potencial y virtual a lo concreto, efectuando, así, el proceso descendente. Esto lo realizamos mediante la determinación.

Para Coseriu las palabras, los signos lingüísticos no son reales, son virtuales: «los nombres que integran el saber lingüístico no son 'actuales', sino 'virtuales'; no significan 'objetos' sino 'conceptos'» (1982, 293).

\footnotetext{
11 Para Ortega y Gasset, igualmente, la metáfora y la poesía son un acto del conocer que se hace en el momento de hacer la metáfora, lo que es no más que la utilización de los medios de la lengua para la creación de un sentido. Dice al respecto: «la poesía es un modo de conocimiento, o dicho con otras palabras $[\ldots]$, lo dicho por la poesía es verdad» $(2001,89)$.
}

Odisea, $n^{\circ} 3,2003$ 
Los conceptos valen para aplicarlos a todo, para estructurar lo informe, lo innómine. Pero no representan a aquellas cosas concretas en las que se desenvuelve el hablante. El hablante se vale de muchos medios para designar aquello que quiere. Uno de ellos es el contexto y la situación, de aquí que ambos medios se hagan lingüísticos. Una expresión no es tal si no está incardinada en un contexto y en una situación. Pero el medio, lingüístico por excelencia, del que nos valemos para aplicar la esencia que hemos atribuido a lo seleccionado, para realizar el proceso descendente, es la determinación. Ésta es un conjunto de operaciones cognoscitivas que tienen por misión orientar los signos a las cosas reales. La determinación pertenece a la técnica general del hablar, y es un procedimiento cognoscitivo:

Para transformar el saber lingüístico en hablar — para decir algo acerca de algo con los nombres - es, pues, necesario dirigir los signos respectivos hacia los objetos, transformando la designación potencial en designación real (denotación) $(1982,294)$.

Se trata de transformar lo que ya hemos elaborado de alguna manera aplicándole intuitivamente una esencia, se trata de modificar los propios contenidos de conciencia y hacerlos que designen lo real:

Ahora bien, «actualizar» un nombre es, precisamente, ese orientar un signo conceptual hacia el ámbito de los objetos. O, más estrictamente, la actualización es la operación mediante la que el significado nominal se trasfiere de la «esencia» (identidad) a la «existencia» (ipsidad), y por la cual el nombre de un «ser» [...] se vuelve denotación de un «ente» [...], de un «existencial» al que la identidad significada se atribuye por el acto mismo de la denotación (íbidem).

Y lo mismo que se han creado las esencias libremente, libremente se vuelven esas esencias hacia lo real. La determinación es acto del conocer, acto libre, acto de transformación de elementos creados en la conciencia del hablante. La lengua no sólo nos suministra los instrumentos del decir y sus esquemas, sino también los instrumentos para hablar y conocer. La determinación no es una operación única. Coseriu distingue cuatro tipos de operaciones dentro de la determinación nominal: la actualización, la discriminación, la delimitación y la identificación.

La justificación que a todas estas operaciones nos da Coseriu pone de manifiesto, una vez más, que se trata del conocer:

Las operaciones que constituyen la determinación aseguran [...] una de las posibilidades fundamentales del hablar: la de referirse sin equivocación a lo actual y particular con signos que de por sí, en el «acervo idiomático», son virtuales, en su mayoría universales, y a menudo equívocos [...]. [L]a determinación asegura simplemente el empleo de la lengua: la integración lingüística entre un conocer actual y un saber anterior $(1982,308)$.

Todo lo creado en la lengua no tiene justificación real. Es una actividad libre, cognoscitiva, hasta tal punto que ésta supera a la técnica del hablar histórico:

Odisea, $n^{\circ} 3,2003$ 
Pero hay algo más importante aún: por un lado, el hablar no emplea todo lo que la lengua puede ofrecerle para una determinada circunstancia y, por el otro, el hablar no sólo emplea la lengua, sino que también la supera, puesto que el conocer supera constantemente lo sabido (ibidem)

El conocer supera siempre a la lengua. La explicación de ésta ha de ser hecha, pues, en función del propio conocer, y es lo que hace Coseriu con su teoría del hablar.

\section{BIBLIOGRAFÍA}

Coseriu, Eugenio. 1952. Sistema, norma y habla. Montevideo.

—1977 (1971). Tradición y novedad en la ciencia del lenguaje: estudios de historia de la lingüística. Madrid: Gredos.

—1981 (1977). Principios de semántica estructural. Madrid: Gredos.

—1982 (1962). Teoría del lenguaje y lingüistica general. Madrid: Gredos.

—1985b (1977). El hombre y su lenguaje: estudios de teoría y metodología lingüistica. Madrid: Gredos.

-1985b. «Linguistic Competence: What is it Really?». The Modern Language Review, 80, 4. XXV-XXXV.

—1986a (1951). Introducción a la lingüística. Madrid: Gredos.

-1986b (1973). Lecciones de lingüistica general. Madrid: Gredos.

—1987 (1978). Gramática, semántica, universales: estudios de lingüística funcional. Madrid: Gredos.

-1988 (1957). Sincronía, diacronía e historia: el problema del cambio lingüistico. Madrid: Gredos.

— 1990. «Semántica estructural y semántica cognitiva». Homenaje al Profesor Francisco Marsá/Jornadas de Filología. Colecció homenatges. Universidad de Barcelona: 239-282.

-1992 (1988). Competencia lingüística: elementos de la teoría del hablar. Madrid: Gredos.

Di Cesare, Donatella. 1999. Wilhelm von Humboldt y el estudio filosófico de las lenguas. Traducción de Ana Agud. Anthropos

Heidegger, Martín. 1970. Carta sobre el humanismo. Madrid: Taurus Ediciones, S. A. -2002 (1944). El ser y el tiempo. Barcelona: RBA Coleccionables.

Humboldt, Wilhelm von. 1990 (1836). Sobre la diversidad de la estructura del lenguaje humano y su influencia sobre el desarrollo espiritual de la humanidad. Traducción de Ana Agud. Madrid: Anthropos y Ministerio de Educación y Ciencia.

Ortega y Gasset, José. 1971 (1935). Historia como sistema. Madrid: Austral. 
-1982 (1958). Goethe - Dilthey. Revista de Occidente en Alianza Editorial.

-1989 (1960). Origen y epílogo de la filosofía. Revista de Occidente en Alianza Editorial.

-1992 (1984) ¿Qué es conocimiento?. Revista de Occidente en Alianza Editorial.

-1996 (1979). Sobre la razón histórica. Revista de Occidente en Alianza Editorial.

-1997 (1940). Ideas y creencias. Revista de Occidente en Alianza Editorial.

-1999 (1966). Unas lecciones de metafisica. Revista de Occidente en Alianza Editorial.

-2001 (1957). El hombre y la gente. Revista de Occidente en Alianza Editorial.

-2002 (1939). Meditación de la técnica y otros ensayos sobre ciencia y filosofía. Revista de Occidente en Alianza Editorial.

-2003 (1966). Europa y la idea de nación. Revista de Occidente en Alianza Editorial. 